\title{
Nanomedicines can Offer Improved Therapeutic Efficacy through Various Parenteral Routes of Administration
}

\section{Balabathula P*}

Plough Center for Sterile Drug Delivery Systems, University of Tennessee Health Science Center, Memphis, Tennessee, USA

*Corresponding author: Balabathula P, Plough Center for Sterile Drug Delivery Systems, University of Tennessee Health Science Center, Memphis, Tennessee, USA, Tel: 001-901-448-2349; E-mail: pbalabat@uthsc.edu

Rec date: Feb 25, 2016; Acc date: Feb 26, 2016; Pub date: Feb 29, 2016

Copyright: ( 2016 Balabathula P. This is an open-access article distributed under the terms of the Creative Commons Attribution License, which permits unrestricted use, distribution, and reproduction in any medium, provided the original author and source are credited.

\section{Editorial}

For life threatening diseases such as infections, the standard drug treatment strategy is to get as much antimicrobial drug as possible to the pathogen as fast as possible to obtain an effective pathogen kill rate [1]. Therefore the route of drug administration is often an injectable true solution because oral administration is often variable in sick patients or physical chemical properties of drug product simply to not allow acceptable bioavailability [2,3]. In critical patients, intravenous administration with an initial bolus dose followed by an infusion to maintain killing concentrations is often the norm [4]. The assumption of this approach is that if sufficient concentrations of the drug are maintained in the blood stream enough of the antimicrobial agent is being "pushed" to the infection site to produce an adequate kill rate.

In contrast, particulate nanomedicines are designed to overcome specific problems in drug delivery. For example, amphotericin B solution has significant renal toxicity and administration problems [5]. Most toxicities are dose related. If killing effectiveness can be raised, the total dose administered can be cut, hopefully avoiding or ameliorating some of the toxicity. In previous studies from our laboratories with highly toxic anticancer agents, formulating the drug into a nanomedicine increased killing efficiency and allowed for a $20 \%-30 \%$ reduction in dose as compared to the non nanomedicine formulations [6]. Several properties contribute to this effect. First, with nanomedicines the dosage form can be targeted to the site of action either passively or actively (or both simultaneously) so that the drug substance is not diluted throughout the body to tissues that do not need the drug $[7,8]$. For nanomedicines, the blood concentration may not be indicative of the concentration needed for treatment. Rather the drug substance concentration at the intended site is more important. Second, nanomedicines can be optimized so the drug has an extended release profile locally over several days. Third, a diagnostic agent in the nanomedicine particles should allow for a more accurate diagnosis as to the spread of the infection [9]. Hopefully, monitoring of subsequent doses will also allow serial image monitoring of the recovery process. This should allow the attending physicians to better monitor and change dosing regimens thereby producing a more controlled therapeutic response. Fourth, nanomedicine particulates often avoid molecule focused active transport body systems that eliminate drugs in true solution from the body $[8,10]$. Drug resistance by this mechanism is a fairly common problem with some critical drugs and is not easily overcome. However, most drug exotransporters work on molecular drugs in true solution, not on significantly larger particulate nanomedicines. Nanomedicine particles are considered to be too large for molecular transporters.
With colloidal nanomedicines, size and surface charge matters. Usually internal nanomedicines for parenteral use are in the range of $200 \mathrm{~nm}$ or smaller $[7,10]$. As size decreases effectiveness can increase. Part of this is due to vascular permeability, Biological barriers such as vasculature become much more porous when inflammation is present. This allows small nanomedicine particles to pass onto tissues and have significant penetration to the infection site. This allows for potential intravenous, intramuscular and subcutaneous routes of administration. Since diseases like invasive fungal infections include infections of the CNS and lungs, the delivery system properties are important. With fungal pneumonias it may be possible to administer nanomedicine particles by nebulization both as a nontargeted and target product. Concurrent intravenous administration may also be warranted.

\section{References}

1. Balabathula P (2015) Development and evaluation of amphotericin B loaded iron oxide nanoparticles for targeted drug delivery to systemic fungal infections.

2. Albengres E, Le Louët H, Tillement JP (1998) Systemic antifungal agents. Drug interactions of clinical significance. Drug Saf 18: 83-97.

3. Sharma RR (2010) Fungal infections of the nervous system: current perspective and controversies in management. Int J Surg 8: 591-601.

4. Jackson A, Van der Horst C (2012) New insights in the prevention, diagnosis, and treatment of cryptococcal meningitis. Current HIV/AIDS Reports 9: 267-277.

5. Burgess BL, He Y, Baker MB, Luo B, Carroll SF, et al. (2013) NanoDisk containing super aggregated amphotericin B: a high therapeutic index antifungal formulation with enhanced potency. International Journal of Nanomedicine 8: 4733-4743.

6. Divi MK (2007) Development and evaluation of brain tumor targeted liposome delivery system for paclitaxel. The University of Tennessee Health Science Center.

7. Bhattacharjee H, Balabathula P, Wood GC (2010) Targeted nanoparticulate drug-delivery systems for treatment of solid tumors: a review. Ther Deliv 1: 713-734.

8. Jabr-Milane LS, van Vlerken LE, Yadav S, Amiji MM (2008) Multifunctional nanocarriers to overcome tumor drug resistance. Cancer Treat Rev 34: 592-602.

9. Wahajuddin, Arora S (2012) Superparamagnetic iron oxide nanoparticles: magnetic nanoplatforms as drug carriers. Int J Nanomedicine 7: 3445-3471.

10. Peer D, Karp JM, Hong S, Farokhzad OC, Margalit R, et al. (2007) Nanocarriers as an emerging platform for cancer therapy. Nat Nanotechnol 2: $751-760$. 